\title{
Enhancing Microburst Pedagogy: Visual Clues for Aviators
}

\author{
Mary M. Snow and Richard K. Snow \\ Embry-Riddle Aeronautical University
}

\begin{abstract}
A particularly uncompromising type of wind shear results from the meteorological phenomenon known as the microburst. This paper addresses the preliminary phase of an ongoing longitudinal study designed to enhance microburst pedagogy. An initial survey has been distributed to aviation faculty members at universities across the United States to collect data on the length of time they allot to teaching microburst recognition skills. These educators have been introduced to graphics that, it is hypothesized, will make their treatment of the topic more enduring. Subsequent data will reveal whether the use of these graphics significantly increased the time spent teaching microburst recognition and avoidance.
\end{abstract}

\section{INTRODUCTION}

As the primary cause of fatalities in aviation, wind shear and its related hazards should be emphasized in aviation and aviation weather curricula. In commercial aviation alone, more than 650 fatalities resulted from wind shear during the past two decades, thus causing wind shear to be named the leading killer in aviation (Miner, 1997). Microbursts cause severe directional and speed shear affecting the entire range of aircraft from single engine planes to wide body jets. If caught directly below a microburst, severe vertical wind shear results. When horizontal winds change more than 15 knots or there are vertical changes of more than 500 feet per minute, wind shear is classified as severe (Holden, 2000).

Understanding the atmospheric dynamics leading to the development of a microburst as well as recognizing the clues that are apparent on the landscape when a microburst is occurring nearby should decrease the chance of the occurrence of another aviation mishap caused by this phenomenon. However, if a pilot inadvertently becomes caught in the grip of a microburst, there is no substitute for the proper escape maneuver. Since the pilot has only 5 to 15 seconds to decide upon and execute the correct procedure (Miner, 1997), instruction of such a maneuver should be clear, thorough, and repeated.

When educators incorporate into their curricula an understanding of the weather conditions leading to a microburst; the recognition of features resulting from a microburst; and the instruction of an effective escape maneuver; the chance of surviving these deadly phenomena should be enhanced. This study is designed to extend the time microbursts are discussed in aviation courses by making available graphics depicting the ground clues that pilots can recognize from the air.

\section{DISCOVERY OF THE MICROBURST}

Dr. Theodore Fujita, originator of the F0F5 tornado scale, was the first to coin the term microburst (Caracena, Holle \& Doswell, 1990). On June 24, 1975, Eastern Airlines' Flight 66 crashed on approach to JFK International Airport in New York injuring 12 people and killing 112 (Wilson \& Wakimoto, 2001; Rosenfeld, 1995). In addition to an analysis of the aircraft's flight data recorders, various pilot reports, and the airport's anemometer, Fujita conducted aerial surveys of the damage from the storm that occurred during the crash of Flight 66 . He noticed a starburst, or radial, pattern created by the downed trees in the area. Fujita recognized that same radial pattern from the previous year when he had viewed the damage following the super tornado outbreak on April 3-4, 1974 (Wilson \& Wakimoto, 2001; Rosenfeld, 1995).

During that jumbo outbreak which was spawned from three almost parallel squall lines, 148 tornadoes with winds exceeding 260 m.p.h. spun from Mississippi to Michigan, killing 309 and injuring 5000 people (Rosenfeld, 1995). Dr. Fujita, along with University of Oklahoma and NOAA's National Severe Storms Laboratory meteorologists, took 3,600 color photographs of the damage that Fujita called a "gold mine" of clues pertaining to severe weather (Rosenfeld, 1995). From 1975-1978, Fujita engaged in an 
aerial photography endeavor during which corn fields and forests were photographed extensively from low-flying Cessna aircraft (Fujita, 1978).

Along with the swirling patterns typifying the twisting motion of tornado vortices, Fujita noticed that strange radial pattern of uprooted trees suggesting the lack of rotation and indicating, rather, the presence of powerful straight-line winds that typify downbursts (Wilson \& Wakimoto, 2001). The idea of a strong fluid jet surge that descends from the base of a cumulonimbus cloud and bursts outward upon striking the ground, which acts as a flat plate had been put forth as early as 1949 (Wilson \& Wakimoto, 2001). Byers and Braham (1949) described these processes of fluid dynamics in their paper to the United States government following their work on the Thunderstorm Project from 1946-1947. However, meteorologists were slow to accept the concept proposed by Byers and Braham (Wilson \& Wakimoto, 2001).

As the mentor of Ted Fujita, Byers suggested that Fujita call the phenomenon causing the radial pattern of damage a downburst. Later, Fujita differentiated between the larger macroburst ( $>4 \mathrm{~km}$ across) and the smaller in diameter $(<4 \mathrm{~km})$ microburst (Wilson \& Wakimoto, 2001). Ted Fujita is also the originator of the term "bow echo" for the crescent-shaped radar signature of those mesoscale systems that are indicative of powerful thunderstorms. The half-moon signatures are approximately 25-75 miles in length and can last for several hours. With the bow pointing in the direction of the storm's movement, these phenomena alert meteorologists to the type of storms that have the potential to produce tornadoes and microbursts (Grenci, 1997).

There is no question that Fujita's keen observation skills, including his uncanny ability to piece together fragments of data, saved countless lives. The small-scale diverging wind feature that Fujita identified as the microburst poses an extreme hazard to aviation safety. According to Wilson and Wakimoto (2001, p. 49), "(t)he subsequent research on this wind shear event and transfer of this knowledge into the aviation community have benefited the whole of society and must be considered one of the major, rapid payoff, success stories in the atmospheric sciences." The task at hand is to relay this valuable information to all pilots.

\section{CAUSAL MECHANISMS OF THE MICROBURST}

A microburst is an extremely powerful, concentrated downdraft from thunderstorms and convective showers (Fujita \& Caracena, 1977). Microbursts are small-scale phenomena, both temporally and spatially. The intense downdraft lasts on average from only 5-7 minutes and is typically less than two miles wide (Chandler, 1992). The mature thunderstorm is marked by the presence of strong updrafts and downdrafts. However, when several factors coincide, the downdrafts can become especially powerful. Associated with cumulonimbus clouds, the microburst "splashes" into the ground causing winds to radiate outward up to $70 \mathrm{~ms}^{-1}$ (Figure 1) (Geerts, 1999).

As the "fair weather" cumulus cloud builds upward into a cumulus congestus, a towering cumulus, and perhaps into the cumulonimbus, drier and cooler ambient air is entrained. Some of the moisture droplets and/or ice crystals within the cloud evaporate rapidly in the presence of this drier air. Evaporation is a cooling process since heat energy is absorbed from the environment to convert liquid water into water vapor. This cooling process initiates the downdraft inside the cumulonimbus cloud. As the downdraft begins to descend through the cloud, so do raindrops. The falling raindrops create a drag on the air surrounding them, also contributing to the power of the downdraft. Additionally, as rain falls from the bottom of the cloud, it enters an unsaturated region below. The evaporation of some of the raindrops in the unsaturated air further accelerates the downward force. In the arid and semi-arid regions of the western United States, the combination of high cloud bases and very dry air often cause all of the rain exiting the bottom of a cloud to evaporate as virga, the presence of which can indicate a microburst. 




Figure 1. Conceptual Vortex Ring Model of a Microburst (Cross Section)

\section{MICROBURST MODELING AND DETECTION}

Weather theory can be used to simulate microburst activity. The relationship between source strength, uniform wind, and rotational strength are determined by using potential flow theory. The theory is then applied to microburst simulation (Rinehart, Borho, \& Curtiss, 1995). Likewise, simulated mid-latitude continental cumulus convection yields statistics to enable detailed analyses of updrafts and downdrafts $(\mathrm{Xu}$ \& Randall, 2001).

Microbursts that are simulated in the laboratory have been used to investigate low-level wind shear and the development of both airborne and ground-based systems for detecting wind shear (Alahyari \& Longmire, 1995). Wind shear detection systems have evolved from the prototype SURFWAS, for Surface Wind Shear Alert System, to the TDWR, or Terminal Doppler Weather Radar system that is currently in use (Liu, Golborne \& Bun, 1998; Hughes, 1994). The SURFWAS was an economical, portable alternative to the FAA's LLWAS, or Low-Level Wind Shear Alert System (Liu, Golborne \& Bun, 1998).

Following the crash of a DC-9, USAir Flight 1016, at Charlotte in the summer of 1994, the FAA hastened the installation of 47 TDWR systems at airports across the Untied States (Keirnan, 1995; Hughes, 1994; Phillips, 1994). The DC-9 had on board the Honeywell Wind 
Shear Detection System that failed to alert the crew to the problem. Crewmembers stated that the wind shear on their approach for runway $18 \mathrm{R}$ "exceeded any they had previously experienced or were trained to cope with" (Phillips, 1994, p. 30).

Unlike ATC radar systems, the TDWR is not located at airports but 8-12 miles away. The unmanned TDWR surveys meteorological conditions over the approach paths to runways, and it transmits to ATC automated alerts when airflows associated with gust fronts, wind shear, or microbursts are detected. The algorithms for detecting adverse wind conditions have a $90 \%$ probability of detecting a microburst (Nordwall, 1996). Indeed, TDWR is credited for the detection of 85 microbursts at Washington National Airport during the summer of 1996 alone (Serafin \& Wilson, 2000).

The FAA plans to install TDWR near 47 large airports in the United States. Since mid1999, 38 TDWRs have been commissioned, two are used for support and training, and five more are in varying stages of development (Serafin \& Wilson, 2000). The FAA's TDWR system was devised to supplement the Weather Surveillance Radar-1988 Doppler (WSR-88D) network and has made remarkable improvements in the detection of severe storms (Vasiloff, 2001). Addressing the need for further technological development, May (2001) demonstrates the limitations of dual Pulse Repetition Time (PRT) radars and their distortions of the signatures of microbursts and mesocyclones.

Recent strides in aviation weather simulation training include the Weather Environment Simulation Technology (WEST), a three-dimensional, virtual reality software that provides very convincing weather scenarios. WEST creates a grid of individual weather elements from actual radar information and satellite imagery. Flying into a wet microburst, for example, the pilot can actually see the vortex while acquiring data on wind direction and speed. While pilot training simulators teach how to recover from wind shear, WEST trains people to recognize the visual signature and avoid a dangerous microburst situation (Godar, 1996).

A recent study (Lazarus, Shapiro \& Droegemeier, 1999) shows that the analysis of a
Single-Doppler Velocity Retrieval (SDVR) technique permits unobserved wind directions to be determined. This technique has been used to retrieve microburst winds. More recently, thrust vectoring (TV) has been introduced (Visser, 1999). This technology has the capability to deal with the loss of control that can accompany severe wind shear. As the name implies, TV enables the propulsion provided by a jet engine to be redirected to a desirable angle with respect to the body of the aircraft to improve wind shear recovery. Originally used by the military, the technology has been proposed to improve the safety of commercial jet aircraft (Gal-Or, 1994).

Other new technologies include Raytheon's Integrated Terminal Weather System (ITWS), which the FAA is preparing to evaluate at Kansas City Airport and others. This system produces real-time data and is expected to provide short-term insights of wind shear and microburst detection and prediction (Nordwall, 2001). Similarly, Unysis has been testing a Microburst Prediction Radar (MPR) at the Memphis Airport following previous experiments in Orlando and Denver ("Unysis Tests," 1994).

Also in progress, a Geostationary Environmental Satellite (GOES) sounder is being used to assess downburst potential from vertical profiles of temperature and moisture up to the 300 mb level (Ellrod et al., 2000). A quite innovative small robotic aircraft, the Aerosonde, has been designed to monitor weather conditions. So far, the Aerosonde has survived more than $6 \mathrm{~g}$ turbulence after flying into a microburst (Holland, Webster, Curry \& Tyrell, 2001).

DC-10 Captain Frank Tullo (1999), with Continental Airlines, reports that during the past two decades, "wind shear training aid" has increased knowledge of this weather hazard. In conjunction with wind shear warning devices on board aircraft, Doppler radar near airfields, and knowledge of the escape procedure, pilots can manuever their aircraft away from microbursts. However, small planes are not equipped with such detection devices, and far too few airports have the Doppler systems in place. With all the accuracy and promise that TDWR offers, some people liken it to a safety net with holes that are too big. 


\section{MICROBURST INDUCED AIRCRAFT CRASHES}

Microbursts are especially dangerous to aircraft on takeoff or landing because of low altitudes and lack of space and time to recover. In addition to the USAir Flight 1016 accident in 1994 at Charlotte and the Eastern Flight 66 at JFK in 1975, microbursts have been associated with numerous crashes of commercial passenger jets. Since 1960, microbursts are believed to have caused at least 30 airliner crashes and more than 500 deaths (Chandler, 1992). Probably the bestknown accident caused by a microburst occurred at Dallas-Fort Worth in 1985 when Delta's Flight 191, a Lockheed L-1011, crashed on approach killing 137 people (NTSB, 1986; Chandler, 1992).

Because of the dynamics of a microburst, an aircraft might first encounter a powerful headwind and gain an enormous amount of lift. An unsuspecting pilot on final approach naturally pitches the nose downward and reduces power to descend to the proper glide slope. Soon thereafter, a powerful tailwind might be encountered, which would dramatically reduce lift and has caused aircraft to crash short of the runway. When pilots are better prepared to recognize microbursts and their associated wind shear, we can better avoid these potentially fatal weather hazards.

\section{VISUAL CLUES FOR PILOTS}

In addition to the technologies that might or might not be available to the general aviation pilot, the ability to "read" the landscape for clues of microburst activity is an invaluable skill. There are excellent microburst graphics available for educators and pilots (i.e. Caracena, Holle \& Doswell, 1990). While many of these photographs have been copyrighted, most of the photographers are willing to share their work without fees for educational purposes.

Certain features of microburst activity are easily recognizable from the air. For example, there is typically a characteristic "foot" at the base of a rain shaft. As the downburst strikes the ground and pushes the rain outward in all directions, a bulge in the rainshaft near the surface renders these winds visible. Subsequently, a "precipitation curl" rises upward marking the "outflow boundary" of the "vortex ring" at the far edges of the microburst (Figure 1). This rising air can cool to the dew point, and scud clouds might form beneath the towering cumulus.

Also visible is the rapid expansion of that vortex ring as the winds can travel at speeds in excess of $100 \mathrm{kts}$, often blowing rain ahead of it. These features can alert the pilot to a "wet microburst" in the humid east of the United States. When downbursts occur in the semi-arid west, a "dry microburst" becomes visible by

blowing dust, debris, and topsoil at the surface and the presence of a dissipating rainshaft extending from the base of a cloud, indicating virga aloft.

Adding complexity to the task of landing an aircraft are the multiple vortices that exist in the divergent winds of the microburst. As the powerful "straight line" winds race across the landscape, turbulent eddies form within. A pilot on approach or take off who successfully deals with a sudden $80 \mathrm{kt}$ headwind could encounter severe directional shear before reaching the center of the microburst. Understanding the internal dynamics as well as the external manifestations associated with the microburst should enhance maintaining control of the plane, or better yet, encourage avoidance.

\section{METHOD}

The long-term purpose of this study is to make pilots more self-reliant while they are flying in severe weather conditions. When the time between an actual threat and a reasonable response to that threat is minimized, safety is improved. A necessary ingredient is to recognize the threat. When the visual clues provided by the landscape are learned and practiced, pilots can bypass the time needed to have radar returns analyzed and warning systems activated. Any time saved could make a difference in the number of fatal crashes due to the loss of control caused by a microburst.

The short-term goal of this research is to increase the amount of time that microbursts are addressed in aviation curricula. The resource used to increase this time is a valuable microburst handbook available on the worldwide web. The 
researchers firmly believe that the quality of the content of microburst lectures is extremely important and will be greatly augmented by these graphics as well. However, the immediate focus will be on the quantity of time devoted to the topic of microbursts. It is hypothesized that the time spent addressing microbursts in the classroom will be significantly increased when visual clues are incorporated into the curricula of educators in the field of aviation. By significant, the researchers are referring to at least a $25 \%$ increase in time spent on microbursts.

A six-question survey has been sent out electronically to professors at institutions with aviation programs to determine whether they teach microbursts, in which courses they teach microbursts, and how much time is presently allotted the topic (Appendix A). The participants also were asked whether they thought that graphics would help them in microburst pedagogy. They were supplied with a web site that includes 33 visual aids the preponderance of which are extraordinarily dynamic photographs of actual microbursts and their associated physical features.

During the Fall 2002 semester, another electronic message will be sent to the participants reminding them of the web site and suggesting that they use some or all of the graphics in class. If the professor does not have access to the Internet in class, the photos can be downloaded onto a disk, or printed as a hard copy from which transparencies could be made. At the end of the Fall 2002 semester a follow-up survey will be sent to the respondents. The follow-up survey will be designed to determine whether the supplied visual clues served to increase the time that those educators spent teaching microbursts. The data will be analyzed to determine whether that amount of time increased, and if so, whether the increase was significant.

\section{SUMMARY}

It is anticipated that by incorporating into microburst curricula the graphics that have been and will continue to be provided, educators will increase the time they spend discussing microbursts in class. It is also anticipated that the amount of time will be increased significantly $(>25 \%)$. While this is the variable that can be measured quantifiably, it is also expected that the quality of microburst pedagogy will be enhanced dramatically. Of the 45 participants who have responded thus far, only one stated that he thought that visual aids would not help his discussion of microbursts. The other 44 respondents appear to be very receptive to the idea of introducing into their curricula the photographs taken by the atmospheric scientists and storm chasers. By directing these educators to these invaluable tools, student pilots should acquire a greater understanding of microburst recognition and avoidance. 


\section{APPENDIX A}

Hello:

My name is Mary Snow, and I teach Meteorology at Embry-Riddle Aeronautical University. I have met many of you and explained my research on microbursts.

It would be of enormous help to my ongoing research if you would take a few minutes to answer six short questions about your curricula. Following the questions, you will find an URL that might prove very helpful to you as you teach downbursts and microbursts in your classes.

Many sincere thanks for your time and thought,

Mary Snow, Ph.D.

Department of Applied Aviation Sciences

Embry-Riddle Aeronautical University

600 S. Clyde Morris Blvd.

Daytona Beach, FL 32114

>>>>>>>>>>>>>>>>>>>>>>>>>>>>>>>>>>>>>>>>>>>>>>>>>>>>>>>>>

1. Is teaching downbursts/microbursts a part of your curricula?

2. If not, would you like it to be? (STOP HERE)

3. If so, in which course(s) do you address microbursts (course titles please). 
4. Approximately how long (hours and/or minutes) do you spend teaching microbursts in your course(s).

5. Do you think visual aids are or would be helpful for teaching microbursts?

6. Approximately what percentage of your students are pilots?

Thank you again and enjoy this excellent site!

http://www.nssl.noaa.gov/ doswell/microbursts/Handbook.html 


\section{REFERENCES}

Alahyari, A. \& Longmire, E. (1995). Dynamics of experimentally simulated microbursts.

American Institute of Aeronautics and Astronautics. AIAA Journal, 11, 2128-2133.

Byers, H. R. \& Braham, R. R., Jr. (1949). The Thunderstorm, U.S. GPO, Washington, D.C., $287 \mathrm{pp}$.

Caracena, F., Holle, R. L., \& Doswell, C. A. (1990). Microbursts: A Handbookfor Visual Indentification, NOAA: GPO, Washington, D.C.

Chandler, D. L. (1992). Detecting ill winds, Technology Review, 95, 8-11.

Ellrod, G. P., Nelson, J. P., Witiw, M. R., Bottos, L., \& Roeder, W. P. (2000). Experimental GOES sounder products for the assessment of downburst potential, Weather and Forecasting, 15, 527542.

Fujita, T.T. (1978). Manual of downburst identification for project MNIMROD. SMRP Research Paper 156, U. of Chicago, 104 pp.

Gal-Or, B. (1994). Thrust vector control eyed for passenger aircraft: A novel methodology to combine jet-engine tests with sub-scale proof-of-concept flight tests, International Journal of Turbo and Jet Engines, 11, 1-9.

Geerts, B. (2001). Estimating downburst-related maximum surface wind speeds by means of proximity soundings in New South Wales, Australia, Weather and Forecasting, 16, 261-269.

Godar, K. (1996). Next best thing to controlling the uncontrollable, Research \& Development, 38, $56 \mathrm{H}$.

Grenci, L. (1997). Bow echoes, Weatherwise, 50, 64.

Holden, H. M. (2000). Wind shear and microbursts, Plane and Pilot, 7, 66-71.

Holland, G. J., Webster, P. J., Curry, J. A., \& Tyrell, G. (2001). The Aerosonde robotic aircraft: A new paradigm for environmental observations, Bulletin of the American Meteorological Society, $82,889-901$.

Hughes, D. (1994). FAA speeds installation of TDWR systems, Aviation Week and Space Technology, 141, 28-29.

Kiernan, V. (1995). When planes fall out of the sky, New Scientist, 146, 41-44.

Lazarus, S., Shapiro, A. \& Droegemeier, K. (1999). Analysis of the Gal-Chen-Zhang singleDoppler velocity retrieval, Journal of Atmospheric and Oceanic Technology, 16, 5-18.

Lincare, E. \& Geerts, B. (1999). Microbursts, Retrieved March 15, 2001, from University of Wyoming Web site: http://www-das.uwyo.edu/ geerts/cwx/notes/chap08/microburst.html

Liu, H. T., Golborne, C., \& Bun, Y. (1998). Surface windshear alert system, part 1: Prototype development, Journal of Aircraft, 35, 422-428.

May, P. (2001). Mesocyclone and microburst signature distortion with dual PRT radars, Journal of Atmospheric and Oceanic Technology, 18, 1229-1233.

Miner, T. H. (1997). Judgement and wind shear. Flying Safety, 53, 18-22.

Nordwall, B. D. (1996). "Noisy" utilities interrupt TDWR, Aviation Week \& Space Technology, $145,79-82$.

Nordwall, B. D. (2001). The FAA is preparing to conduct an evaluation of Raytheon's integrated terminal weather system, Aviation Week and Space Technology, 155, 84-86.

NTSB examines conditions leading to Dallas/Ft. Worth accident, Aviation Week and Space Technology, 125, 168-169.

Phillips, E. H. (1994). Crash probe focuses on microbursts: Crash of USAir DC-9 at Charlotte, N.C., Aviation Week and Space Technology, 141, 30.

Rinehart, R. E., Borho, A., \& Curtiss, C. (1995). Microburst rotation: Simulations and Observations, Journal of Applied Meteorology, 6, 1267-1285.

Rosenfeld, J. (1995). The jumbo outbreak, Weatherwise, 48, 58.

Serafin, R. J. \& Wilson, J. W. (2000). Operational weather radar in the United States: Progress and 
opportunity, Bulletin of the American Meteorological Society, 81, 501-518.

Tullo, F. J. (1999). Let's make better use of simulator time, Aviation Week \& Space Technology, 151, 66-69.

Unysis tests low-cost microburst radar. (1994). Aviation Week and Space Technology, 141, 37-40.

Vasiloff, S. V. (2001). Improving tornado warnings with the Federal Aviation Administration's terminal doppler radar, Bulletin of the American Meteorological Society, 82, 861-874.

Visser, H. G. (1999). Windshear recovery using thrust vectoring, Aircraft Engineering and Aerospace Technology, 71, 329-333.

Wilson, J.W. \& Wakimoto, R. M. (2001). The discovery of the downburst: T. T. Fujita's contribution, Bulletin of the American Meteorological Society, 82, 49-62.

Xu, K. \& Randall, D. A. (2001). Updraft and downdraft statistics of simulated tropical and midlatitude cumulus, Journal of the Atmospheric Sciences, 58, 1630-1649. 